\title{
Segmentation and Morphometry of Histological Sections Using Deformable Models: A New Tool for Evaluating Testicular Histopathology
}

\author{
Miguel A. Guevara ${ }^{1}$, Augusto Silva ${ }^{2}$, Helena Oliveira ${ }^{3}$, Maria de Lourdes Pereira ${ }^{3}$, and \\ Fernando Morgado ${ }^{3}$ \\ ${ }^{1}$ Computer Sciences Faculty, University of Ciego de Avila, Ciego de Avila 69450, Cuba \\ mguevaral@yahoo.com \\ ${ }^{2}$ IEETA, University of Aveiro, $3810-193$ Aveiro, Portugal \\ asilva@ieeta.pt \\ ${ }^{3}$ Biology Department, University of Aveiro, 3810 - Aveiro, Portugal \\ \{holiveira, lpereira, fmorgado\}@bio.ua.pt
}

\begin{abstract}
This paper presents a tool that uses image segmentation and morphometric methods to evaluate testicular toxicity through the analysis of histological sections of mice testis. The tool is based on deformable models (Snakes) and includes several adaptations to solve important difficulties of histological sections imaging, mainly the low contrast edges between the boundary tissue of seminiferous tubules and the interstitial tissue. The method is designed to produce accurate segmentation and to keep track of tubular identities on images under study. The extracted data can be used straightforwardly to compute quantitative parameters characterizing tubular morphology. The method was validated on a realistic data set and the results were compared with those obtained with traditional techniques. The application of this new technique facilitates measurements allowing assessing a higher number of tubules in a fastest and accurate way.
\end{abstract}

\section{Introduction}

Histopathology is considered the most sensitive endpoint for evaluating testicular toxicology. One of histopathological signs of testicular toxicology is tubular contraction or dilation. Tubular contraction occurs as a result of reduction of fluid secretion and consequently reduction in the overall diameter of the seminiferous tubule. Chlordane is one of the substances that induce a reduction in the diameter of seminiferous tubules [1]. Tubular dilation, with the dilation of tubular lumen may also occur as a result of the increase of the fluid volume in the lumen, or as a consequence of obstruction of fluid flow. For example carbendazim causes obstruction of the ductular system that result in a severe and diffuse dilation of the seminiferous tubules [2]. The dilation of the tubular lumen may be a diffuse change and may not be obvious by microscopic observation [3], so quantitative analysis measuring tubule diameters or tubule area may be a sensitive way to detect tubule dilation.

In this paper, we present a new technique, motivated by the desire to study histological testicular sections for evaluating testicular toxicology. Our method is 
based on deformable models (snakes) and includes several adaptations to solve important difficulties posed by histological section imaging, mainly the low contrast edges between the boundary tissue of seminiferous tubules and the interstitial tissue. First a median filter and morphological operations are applied to enhance the image contrast and differentiation of objects (seminiferous tubules) from background. Second a local edge detector is applied to obtain an approximation to objects contours. Segmentation is completed using an adapted variant of a gradient vector flow (GVF) model [4], [5]. A smoothed instance of the final contour is then obtained through spline approximation based on the detected edge points. Morphometric parameters such as area, diameter and others are then easily computed. Our method was validated on a realistic data set and the results compared with those obtained with traditional techniques.

In section 2, we describe preprocessing steps designed to enhance the detection of seminiferous tubules, where we introduce a new local edge detector based on the use of local average of differences between pixels and the median of neighborhood pixels. Section 3 recalls the original snake model [6], as well as a useful and popular extension, the GVF model [4], [5], and discusses their applicability to our data. Computation of features and measurements such as diameters and area are described in section 4. In section 5, we show results of our method and briefly present an algorithm prototype to study tubular testicular sections. Section 6 concludes with a short summary of our work.

\section{Preprocessing}

In this study male mice were used. After sacrifice, the left testes were removed for histological studies. Small pieces of testis tissue were fixed in Bouin's fluid, dehydrated and embedded in paraffin wax. Transverse tissue sections with 5 microns thick were made in a microtome (Leitz model 1512) and stained with haematoxylin and eosin $(\mathrm{H} \& \mathrm{E})$. Images were acquired with the Leica IM 100 Image Manager software. The hardware used to capture the images was composed by a Leica DC 200 camera attached to a Leitz Laborlux K microscope. However, our prototype accepts as input any true color image in jpg or bmp format. The difficulty in detecting tubular testicular sections stems for the fact that they can not be distinguished only based on their gray level or gradient values, or using a simple combination of smoothing and edge detector filters. To address these problems we attempted with a combination of noise suppression filter like average and median filter, with different mask sizes and histogram thresholding, followed by binarization and edge tracking [7]. Experiments were also carried out with edge maps detectors [8]. However, these methods fail frequently where tubular sections are out the focal plane or due to background contamination. We propose a new approach that is a combination of median filtering, morphological processing and a new developed edge detector to produce an edge map to be processed by the GVF Snake [4], [5]. 


\subsection{Initial Image Preparation}

The presence of noise in images represents an irrecoverable loss of information. The median type filters are widely used for noise suppression in early stages of a vision system [9], due to the following properties:

- They preserve the ramp edges and boundaries of the objects,

- They suppress impulses of short duration without significantly modifying others components, and,

- They may be implemented easily and fast.

The steps followed in the initial image preparation were: convert the input image I (a true color image) first to a 256 gray level image and then build the median image $\mathrm{M}$, applying the median filter with a mask of size $5 \times 5$.

\subsection{Morphological Operations}

Mathematical morphology is a novel geometry-based technique for image processing and analysis, originally developed to process binary images, based on the use of simple concepts from set theory and geometry such as set inclusion, intersection, union, complementation, and translation [10]. This resulted in a collection of tools, called morphological operators, which are eminently suited for the analysis of shape and structure in binary images [11]. The most well-known of these operators are erosion and dilation. Soon mathematical morphology was extended to grey-scale images. To extend binary morphology to grey-scale images different approaches have been proposed. Our work relied on the threshold set approach, in which a grey-scale image is decomposed in terms of its threshold (or level) sets. To each of these sets one can apply a binary operator, after which the resulting sets can be used to synthesize a transformed gray scale image [11].

A morphological operator when applied to a binary image may be regarded as a binary convolution where the convolution kernel is usually defined within a small mask. This kernel is better known as the operator structuring element, and its size and shape determines the outcome of the operation. Morphological operations apply structuring elements to an input image, creating an output image of the same size. Therefore the choice of size and shape of the structuring element be a major design issue in our image preprocessing strategy.

Image Enhancement. Images under study contain many seminiferous tubules of different sizes that may be touching each other. Due to this, to increase the potential for future object discrimination we use a suitable combination of the top-hat and bottom-hat operations. The top-hat transform is defined as the difference between the original image and its opening. The opening of an image is the collection of foreground parts of an image that fits a particular structuring element. The bottom-hat transform is defined as the difference between the closing of the original image and the original image. The closing of an image is the collection of background parts of an image that fit a particular structuring element. We evaluated structuring elements of different shapes and sizes, obtaining the best results with the octagonal structuring element. A flat octagonal structuring element was created computing the radius of the minimum horizontal diameter in the smallest seminiferous tubule of the image under study. Figure 1b show results obtained after to enhance image. 
The mathematical formulation used to enhance $M$ was:

$\mathrm{E}=\mathrm{A}-\mathrm{B}$ where $\mathrm{E}$ is the enhanced image

$\mathrm{A}=\mathrm{M}+\mathrm{T}$

$\mathrm{B}=\varphi(\mathrm{M})-\mathrm{M}$ bottom- hat of $\mathrm{M}$

$\varphi(\mathrm{M})=\mathrm{M} \bullet \mathrm{K}=[(\mathrm{M} \oplus \mathrm{K}) \diamond \mathrm{K}] \quad$ close $\mathrm{M}$ with the structuring element $\mathrm{K}$ $\mathrm{T}=\mathrm{M}-\gamma(\mathrm{M})$ top - hat of $\mathrm{M}$

$\gamma(\mathrm{M})=\mathrm{M} \circ \mathrm{K}=[(\mathrm{M} \ominus \mathrm{K}) \oplus \mathrm{K}] \quad$ open $\mathrm{M}$ with the structuring element $\mathrm{K}$

$\mathrm{M}$ Median image

K Structuring element

$\ominus$ erode operator

$\oplus$ dilate operator

Objects Differentiation. The image complement in combination with the flood-fill were the operations used for object (seminiferous tubules) differentiation (Fig. 1c). Image complement consists in subtracting each image pixel value from the maximum gray level pixel value supported by the image (in our case 255). Image flood-fill fills holes in the input image. A hole is a set of background pixels that cannot be reached by filling in the background from the edge of the image, in our case, a hole is an area of dark pixels surrounded by lighter pixels.

The mathematical formulation to achieve objects differentiation (image $\mathrm{X}$ ) was the following:

$\mathrm{X}=\left[f f\left(\mathrm{~K}^{C}\right)\right]^{C}$ where $f f$ is the flood - fill operation

$\mathrm{K}^{C}$ image complement of $\mathrm{K} ; \mathrm{K}=\mathrm{E}-\mathrm{E}^{C}$ where $\mathrm{E}$ is the enhanced image .

\subsection{Edge Detection}

Edge detection is a critical step, since edge information is major driving factor in subsequent "snake" performance. As we mentioned before in the initial part of this section different techniques were tested such as a combination of noise suppression by average and median filtering, with different masks and histogram thresholding, followed by binarization and edge tracking [7]. We also tried with edge maps detectors [8], but these methods fail frequently where tubular sections are out the focal plane or due to background contamination. However we found that applying a local median average to images has achieved a more suitable answer to distinguish boundary pixels between objects and background pixels. With a $3 \times 3$ mask size this new filter is a powerful edge detector, which produces a fine edge map contour (Fig. 1d).

The mathematical formulation of our edge detector is the following:

$\mathrm{X}$ input image

$\omega \quad N \times N$ windows center on pixel $\left(i_{0}, j_{0}\right)$

$M=\frac{N-1}{2}$ 
$\varpi$ median of $\omega$

$\mathrm{E}_{\text {map }}$ ouput image

$$
\mathrm{E}_{\text {map }}(i, j)=\frac{1}{(2 M+1)^{2}} \sum_{(k, l) \in[-M, M]}\left|\mathrm{X}\left(i_{0}+k, j_{0}+l\right)-\varpi\right|
$$

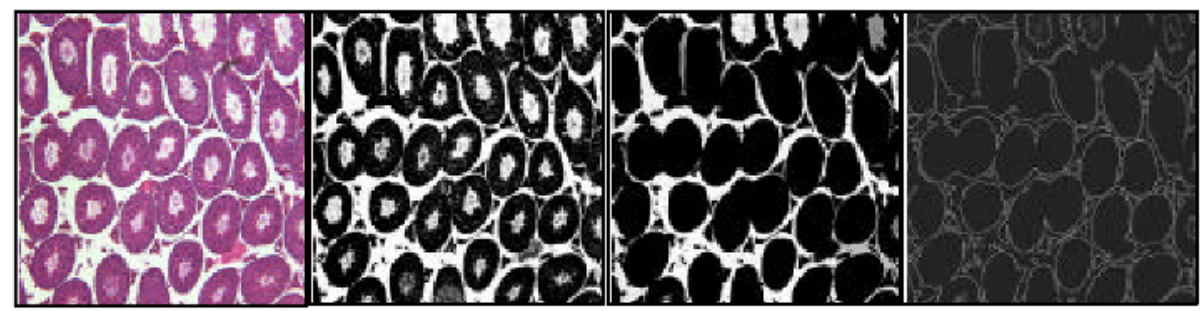

(a)

(b)

(c)

(d)

Fig. 1. (a) Original image. (b) Enhanced image. (c) Tubules differentiation. (d) Local median average.

\section{Deformable Models}

Mathematical foundations of deformable models represent the confluence of geometry, physics, and approximation theory. Geometry is used to represent object shape, physics inflict constraints on how the shape may vary over space and time, and optimal approximation theory make available the formal underpinnings of mechanisms for fitting the models to measured data. Deformable curve, surface, and solid models gained popularity after they were proposed by Terzopoulos for use in computer vision [12] and computer graphics [13] in the mid 1980's. Terzopoulos introduced the theory of continuous (multidimensional) deformable models in a Lagrangian dynamics setting, based on deformation energies in the form of (controlled continuity) generalized splines. The deformable model that has attracted the most attention to date is popularly known as "snakes" [6]. Snakes are planar deformable contours that are useful in several image analysis tasks. They are often used to approximate the locations and shapes of object boundaries in images based on the reasonable assumption that boundaries are piecewise continuous or smooth.

One of the more successful implementation of parametric active contours is the gradient vector flow (GVF) field approach proposed by $\mathrm{Xu}$ and Prince [4], [5]. The active contour that uses the GVF field as its external force is called a GVF snake. The GVF field points toward the object boundaries when is very near to the boundary, but varies smoothly over homogeneous image regions, extending to image border. The main advantages of the GVF field over the original snake model are that it can capture a snake from a long range - from either side of the object boundary - and can force it into concave regions. However, although the GVF model was proven to be superior capturing a snake from a long range from either side of the object boundary, it did not solve the difficulties posed by background contamination and the lost of contrast between seminiferous tubules edges. Therefore, our work was focused to develop a specific approach to enhance contrasts and to improve the edge map of images under study. These processes were described in details in the preprocessing section. We use 
the GVF snake model [4], [5] to complete the segmentation process, which can be summarized as follows: for each edge map ( $\mathrm{E}_{\text {map }}$ ) we computed the GVF field (Fig.

2a). Then in a semiautomatic way are defined polygons. This procedure is carrying out first selecting manually a few edge points from seminiferous tubules. After initial snakes (Fig. 2a) are produced through spline approximation based on the selected polygons. Finally the GVF snake deformation is performed for producing an array of edge point $\varsigma$ (final snakes) with a more accurate segmentation results (Fig. 2b). We used the following parameters values in the snake deformation process: elasticity $(0.05)$, rigidity $(0.0)$, viscosity (1), external force weight (0.6) and pressure force weight (0).

\section{Features}

Morphometric features express the overall size and shape of objects. For these features only the object mask $\mathrm{O}$ and its border $\varsigma$ are needed, not the actual gray scale image [14]. In our case the shape and size were decisive to discriminate seminiferous tubules, allowing to analysis their dilation and contraction on images under study. Traditional methods only take in consideration the smallest horizontal diameter to differentiate tubules. Our proposal introduces the computation of minimum and maximum diameters and area. We use as input the final snake deformation $\varsigma$ (see section 3), which (as was described before) is the array of edge points of seminiferous tubules detected.

Mathematical formulation and computational sequence of measurements is the following:

$\mathrm{O}$ object pixels (seminiferous tubule)

$\varsigma \subset \mathrm{O}$ set of edge pixels, contour of $\mathrm{O}$ (final snake deformation points)

$P_{\varsigma} \in \varsigma$ edge point

$\mathrm{A}=|\mathrm{O}|$ area $=$ number of elements of $\mathrm{O}$

$c i=\frac{1}{\mathrm{~A}} \sum_{(i, j)=p \in \mathrm{O}} i \quad i$ - coordinate of centroid

$c j=\frac{1}{\mathrm{~A}} \sum_{(i, j)=p \in \mathrm{O}} j j$ - coordinate of centroid

$C$ centroid point with coordinates $(c i, c j)$

$d(p 1, p 2)=$ ecludian distance between $p 1$ and $p 2$

$\mathrm{rad}=\min \left(d\left(C, P_{\varsigma}\right)\right)$ minimum radius

$P_{\varsigma_{-} \text {diam }}(0)=P_{\varsigma}$ with $\mathrm{rad} \cap P_{\varsigma} \neq \varnothing$ initial minimum diameter point

$R A D=\max \left(d\left(C, P_{\varsigma}\right)\right)$ maximum radius

$P_{\varsigma_{-} D I A M}(0)=P_{\varsigma}$ with $R A D \cap P_{\varsigma} \neq \varnothing$ initial maximum diameter point 


$$
\begin{aligned}
& P_{\text {diam }}=P_{\varsigma_{-} \text {diam }}(0)+2 * \text { rad } \\
& P_{\varsigma_{-} \text {diam }}(1)=P_{\varsigma} \longrightarrow \min \left(d\left(P_{\text {diam }}, P_{\varsigma}\right)\right) \text { final minimum diameter point } \\
& \text { diam }=d\left(P_{\varsigma_{-} \text {diam }}(1), P_{\varsigma_{-} \text {diam }}(0)\right) \text { minimum diameter } \\
& P_{\text {DIAM }}=P_{\varsigma_{-} R A D}(0)+2 * R A D \\
& P_{\varsigma_{-} \text {DIAM }}(1)=P_{\varsigma} \longrightarrow \min \left(d\left(P_{\text {DIAM }}, P_{\varsigma}\right)\right) \text { final maximum diameter point } \\
& \text { DIAM }=d\left(P_{\varsigma_{-} \text {DIAM }}(1), P_{\varsigma_{-} D I A M}(0)\right) \text { maximum diameter }
\end{aligned}
$$

Finally calculations include an average of area, minimum and maximum diameters of the seminiferous tubules set processed on the image under study (see figure 2c).

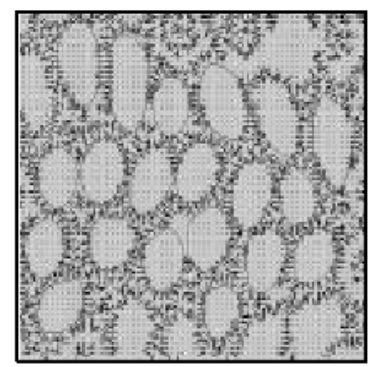

(a)

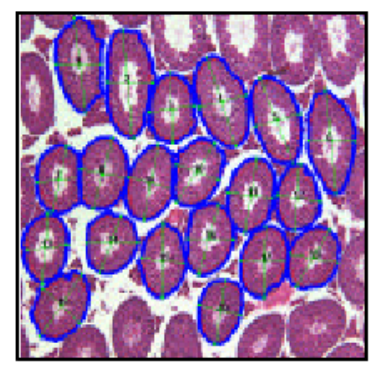

(b)

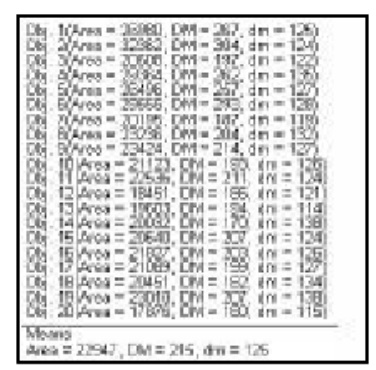

(c)

Fig. 2. (a) GVF and initial snakes (b) Final snake deformation. (c) Micron's measures of each object (seminiferous tubules): area, maximum diameter (DM) and minimum diameter (dm), and the averages of area, maximum diameter and minimum diameter.

\section{Results}

The practical implementation of our technique for evaluating testicular toxicity in histological sections images can be summarized as follows: for each image, we first construct the edge map $\left(\mathrm{E}_{\text {map }}\right)$, based on procedures described in section 2 . Then the GVF field is computed. After in a semiautomatic way polygons are defined base on manual selection of few edge points from seminiferous tubules. The initial snakes then are produced (Fig. 2a) through spline approximation based on the selected polygons. Finally, snake deformation is performed to produce an array of edge point $\varsigma$ (final snakes) with more precise segmentation results (Fig. 2b).

The traditional method for measuring seminiferous tubule diameter is based on measurement of the minimum diameter. However, it is difficult, if not impossible, for the human eye to distinguish which is the minimum diameter, so an approximation must be made. This approximation may lead to a lost of accuracy, mainly in the toxicology studies, because if we can not be sure that we are measuring the same feature it's difficult to compare between different treatments. Our new method allows numerating the tubules measured and provides a range of parameters related to the tubule (e.g. area, minimum and maximum diameter). By the end, we know exactly 
which tubules were measured and the characteristics of each one. By other hand, applying the traditional method, we cannot mark the tubules that are being measured, so the same tubule can be measured twice, and we can not be absolutely sure that the measurement is being made by the minimum diameter.

\section{Conclusions}

We presented a semiautomatic method to segment and to measure histological testicular sections, which in a first step is the result of a correct combination of statistical and morphological operations. Hereafter based on the detected edges the segmentation process is completed using a parametric deformable model. Our technique allows a suitable segmentation of seminiferous tubules in histological section. The ability of our method was demonstrated on an experimental representative data set. This approach will be successful to study quantitatively histological sections of different specimens and can be extended easily to other fields of study such as cell counting and somatic embryos classification among others. Compared with traditional methods, our method computes two new measures: the maximum diameter and the area, and it facilitate measurements, assessing a higher number of tubules in a fastest and reproducible way and minimizing the typical errors.

Acknowledgment. The authors wish to thank to Research Institute of University of Aveiro Project CTS 2002/22 for financial support.

\section{References}

1. Al-Omar M.A., Abbas A.K., Al-Obaidy S.A.: Combined Effect of Exposure to Lead and Chlordane on the Testicular Tissues of Swiss Mice. Toxicology Letters 115(1) (2000) 1-8.

2. Hess R.A. and Nakai M.: Histopathology of the male reproductive system induced by the fungicide benomyl. Histology and Histopathology 15: (2000) 207-2024.

3. Creasy D.M.: Pathogenesis of male reproductive toxicity. Toxicologic Pathology 29: (2001) 64-76

4. Xu, C. and Prince, J.L.: Gradient Vector Flow. A New External Force for Snakes. Proc. IEEE Conf. on Comp. Vis. Patt. Recog. (CVPR), Los Alamitos: Comp. Soc. Press. (1997) 66-71.

5. Xu, C. and Prince, J.L.: Snakes, shapes, and gradient vector flow. IEEE Trans. Image Processing, Vol. 7 (1998) 359-369.

6. Kass, M., Witkin, A. and Terzopoulos, D.: Snakes: Active contour models. International Journal of Computer Vision 1(4) (1988) 321-331.

7. Guevara, M. A., Rodríguez R.: Knowledge-based vision techniques to classify sugarcane somatic embryos. Proceedings $13^{\text {th }}$ ISPE/IEEE International Conference on CAD/CAM, Robotic and Factories of the Future (CARS \& FOF'97). (1997) 388-396.

8. Canny J.F.: A computational approach to edge detection. IEEE Trans. Pattern Anal. Machine Intell. Vol. PAMI-8, (1986) 679-678.

9. Zheng, J., Valavanis, K., and Gauch.: Noise removal from color images, Journal of Intelligent and Robotic Systems, Kluwer Academic Publisher 7: (1993) 257-285.

10. Serra, J.: Image Analysis and Mathematical Morphology. Academic Press, London (1982).

11. Soille, P. Morphological Image Analysis. Springer-Verlag, Berlin (1999). 
12. Terzopoulos, D., Witkin, A. and Kass, M.: Constraints on deformable models: Recovering 3D shape and nonrigid motion. Artificial Intelligence 36(1): (1988) 91-123.

13. Terzopoulos, D. and Fleischer, K.: Deformable models. The Visual Computer 4(6): (1988) 306-331.

14. Rodenacker K., Bengtsson E.: A feature set for cytometry on digitized microscopic images. Analytical Cellular Pathology IOS Press, Vol. 25 (2003). 1-36. 